\title{
Study of dual-phase drive synchronization method and temperature measurement algorithm for measuring external surface temperatures of ethylene cracking furnace tubes
}

\author{
Zhiping Peng ${ }^{1}$ · Jieguang $\mathrm{He}^{1} \cdot$ Yun $\mathrm{Tan}^{2}$ • Delong Cui ${ }^{1} \cdot \mathrm{Qirui}_{\mathrm{Li}}{ }^{1}$ · Jingbo Qiu ${ }^{1}$
}

Received: 2 December 2017 / Accepted: 25 June 2018 / Published online: 28 June 2018

(C) The Author(s) 2018

\begin{abstract}
Currently, the manual method using hand-held infrared temperature measurement instruments for measuring temperatures on the external surfaces of ethylene cracking furnace tubes is highly subjective and is affected by a number of prominent issues, such as the high temperature working environments, which leads to low efficiency and poor measurement accuracy. Hence, an automatic temperature measurement system based on infrared light is designed and realized. In the system, a dual-phase drive synchronization method is proposed to rotate the thermodetector during horizontal movements, thus realizing automatic batch temperature measurements of the furnace tubes. Moreover, a temperature processing algorithm is developed to automatically identify furnace wall and tube surface temperatures, filter out abnormal temperatures and select only high-quality temperature measurements prior to calculating the final result. Real temperature measurement experiments demonstrated that the dual-phase drive temperature measurement system and temperature processing method are effective and efficient. Compared with the traditional manual way, temperatures obtained using the proposed system are more stable and accurate.
\end{abstract}

Keywords Cracking furnace tube - Surface temperature measurement · Dual-phase drive synchronization · Temperature processing algorithm

\section{Introduction}

Generally, cracking technology is one of the key production technologies of ethylene industry [1], so the operating conditions of the cracking furnace directly affect the yield and quality of ethylene [2-4]. The tubes are the most important part of a cracking furnace, and play a role in heating materials and acting as a reactor. Temperature is the main factor influencing cracking furnace tube failure [5] since the tubes are susceptible to corrosion, surface oxidation, coking, mechanical degradation, and distortion due to the high temperature environment. Therefore, one of the most important activities of operating a cracking furnace is monitoring the

Jieguang He

hubice@163.com

1 College of Computer and Electronic Information, Guangdong University of Petrochemical Technology, 525000 Maoming, China

2 College of Mechanical and Electrical Engineering, Guangdong University of Petrochemical Technology, 525000 Maoming, China temperature of furnace tubes by measuring and comparing the temperature of the tube surface with that of the gas outlet [6], referred to as the coil outlet temperature. At present, the most widely used tube surface temperature measurement method is to use a hand-held infrared thermometer for measuring, which locates and measures tube temperature via a hole in the cracking furnace. However, the method presents a number of drawbacks related to the limited observation range of the furnace, hostile temperature measurement environment, and awkward tube locations, which result in difficulties distinguishing between the temperature of the furnace tube surface and furnace wall, poor reproducibility of temperature measurement results, low efficiency, and high labor intensity.

Previous reports on measuring the temperature of cracking furnace tube surfaces or tubular furnaces are limited. Zhou et al. [7] performed simulations and experimental investigations on the simultaneous reconstruction of temperature distributions, absorptivity of wall surfaces, and absorption coefficients of a medium within two-dimensional furnace systems. Furthermore, Masoumi et al. [8] used a dynamic programming technique to develop an optimal 
temperature profile along the reactor of an ethylene cracking furnace. Lou et al. [9] obtained visible thermal radiation images in an oil-fired tunnel furnace using a 2-CCD multi-spectral camera and measured wall surface temperatures based on the radiation images. In a similar way, Cheng et al. [10] used radiation image processing technology to obtain simultaneous measurements of three-dimensional temperature distributions and radiative properties in a gasfired pilot tubular furnace. Zheng et al. [11] developed a distributed parameter model of the tubular reactors in an ethylene cracking furnace using a three-dimensional temperature reconstruction. Moreover, Peng [12] presented a temperature discrimination method and measuring device for distinguishing between ethylene cracking furnace tubes and furnace walls.

Based on the literature above, it can be concluded that the current research and applications mainly focus on adapting existing tube temperature measuring technologies, tools, or theories, and less on the development of new online automation equipment for measuring tubes in batches. Therefore, in this paper, a dual-phase drive synchronization method is proposed as a cracking furnace tube surface temperature measuring device, which successfully rotates the thermodetector during horizontal movement. Furthermore, a temperature processing algorithm is presented to automatically identify the furnace wall and tube surface temperatures, filter out any abnormal temperature, and select only high-quality temperatures, thereby obtaining the final result as the average of selected temperatures.

\section{Structure of temperature measurement system}

The entire system (device) is composed of two parts: the thermodetector and temperature measurement drive platform, as shown in Fig. 1. The control module of the system

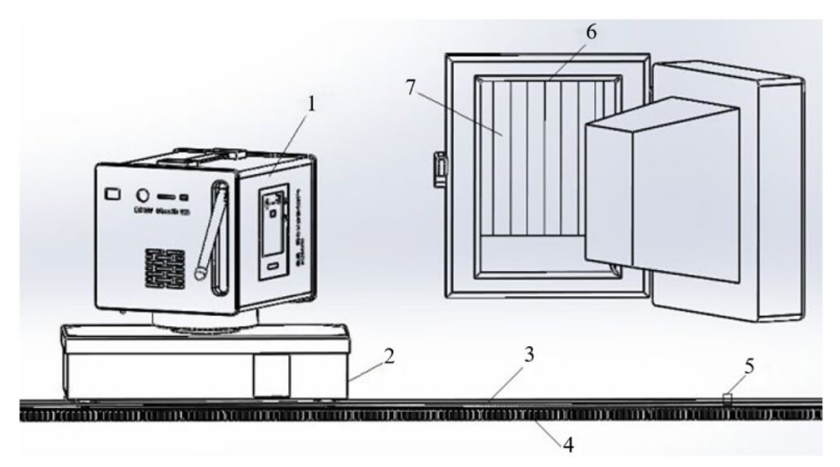

Fig. 1 Schematic diagram of temperature measurement system. 1: thermodetector; 2: temperature measurement drive platform; 3: guide rail; 4: rack; 5: magnet; 6: watching-fire hole; 7: furnace tube is constructed on an ARM cortex-3 STM32 embedded system, which is used to control the dual-phase drive synchronization movement and process measured temperatures. The thermodetector gathers a series of tube surface temperatures without contact via the infrared temperature measurement module, and at the same time, measures the distance between the thermodetector and measured object using the laser ranging module. The temperature measurement drive platform is equipped with a horizontal drive motor and rotary drive motor, which are used to synchronously rotate the thermometer about the horizontal plane as it moves horizontally on the guide rail. Furnace tube temperatures are measured through a watching-fire hole (window) using the thermodetector and the guide rail has grooves on both sides and is furnished with a rack and magnet.

Figure 2 shows the temperature measurement drive platform in detail. Four guide wheels are embedded into the grooves of the guide rail and installed on the baseplate. The horizontal drive motor, rotary drive motor, Hall switch, and optoelectronic switch (through the holder) are also mounted on the baseplate. The horizontal drive motor is connected to gears, which drive the platform forward along the rack. Finally, the rotary drive motor is connected to the rotator, equipped with a baffle.

Figures 3 and 4 illustrate the real temperature measurement system and temperature measurement process applied to an ethylene plant. Dimensions of the thermodetector are $16.5 \times 10 \times 11 \mathrm{~cm}$, and dimensions of the temperature measuring drive platform are $28 \times 12 \times 10 \mathrm{~cm}$. A Panasonic rechargeable lithium battery $(20,400 \mathrm{mAh})$ is installed in the thermodetector and used as the power supply for the whole system. The mobile power source allows temperature measurements to be taken for approximately $4 \mathrm{~h}$ without interruption. The most important components of the thermodetector are the laser range finder (KEYENCE, Japan) and laser temperature probe (Raytek, USA), which are both industrial grade. Due to the high stability and precision of these two

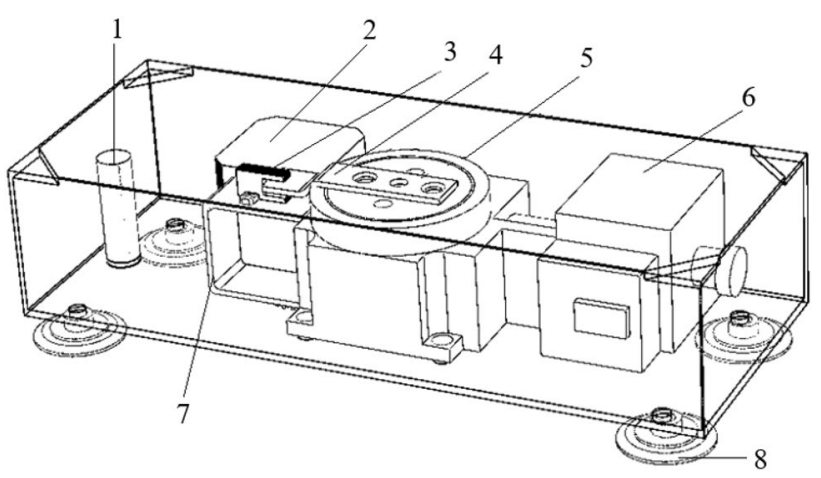

Fig. 2 Schematic diagram of temperature drive platform. 1: hall switch; 2: horizontal drive motor; 3: optoelectronic switch; 4: baffle; 5: rotator; 6: rotary drive motor; 7: holder; 8: guide wheel 


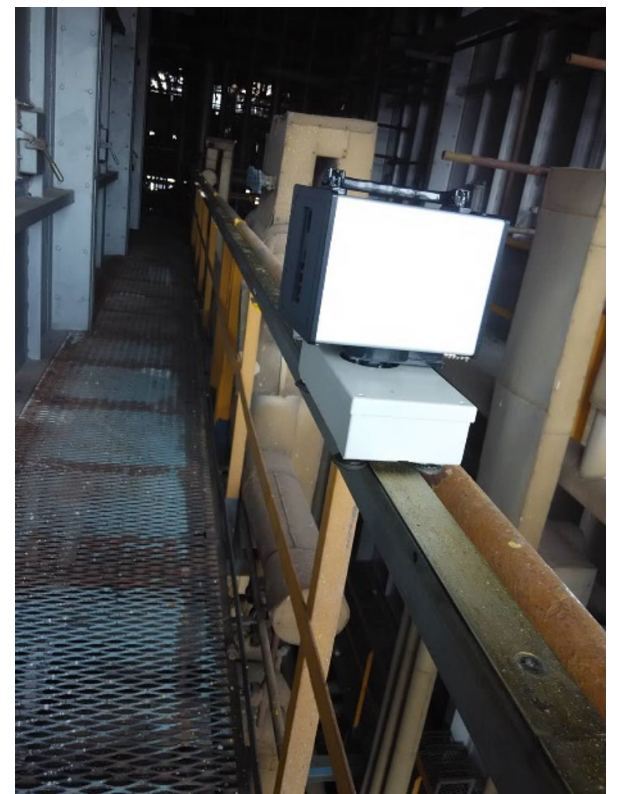

Fig. 3 Real temperature measurement system

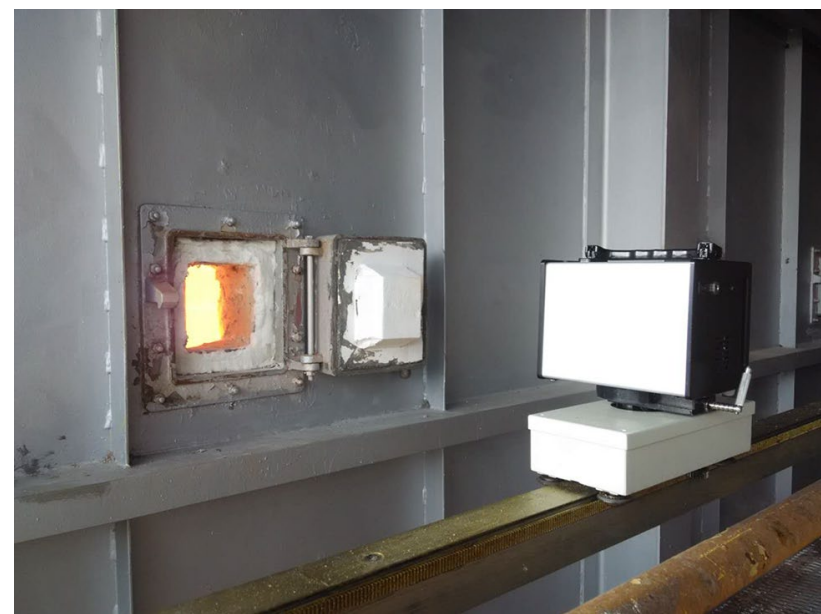

Fig. 4 Temperature measurement process for ethylene cracking furnace tube

instruments, even in high temperature environments, the emissivity of the thermodetector is almost unaffected by the rise in temperature, therefore, the thermodetector is able to accurately measure the temperature of tube surfaces. The diameter of the light spot emitted for measuring the distance or temperature is only $2-3 \mathrm{~mm}$, whereas the diameter of the tube is $20-30 \mathrm{~cm}$. Therefore, to provide a rich source of data for subsequent temperature processing, a series of temperature measurement points are collected on the surface of the furnace tube. Furthermore, the spot does not stray during the measuring process owing to the use of industrial grade range and temperature measurement instruments.
The small size of the system and its capacity to be recharged make it relatively portable. In addition, extra equipment is not required in the temperature measuring field, with the exception of a guide rail, installed on the guard bar outside the cracking furnace.

\section{Dual-phase drive synchronization method for thermodetector}

Since the thermodetector performs bipolar movements, the spot of emitted light also moves. Hence, to evenly scan the tubes through corresponding watching-fire holes, it is necessary to control the position and speed of the emitted light. For this, a dual-phase drive synchronization method is proposed to rotate the thermodetector as it moves along the guide rail.

\section{Steps of dual-phase drive synchronization method}

The following steps also referred to Fig. 5 can be performed to detect temperature using the dual-phase drive synchronization method:

1. Turn on the temperature measurement system.

2. Wait for the measurement signal to be detected.

3. When the signal is detected, the temperature measurement platform is driven by the horizontal drive motor and the platform carrying the thermodetector moves left along the guide rail until the Hall switch and magnet are on the same vertical axis. The thermodetector con-

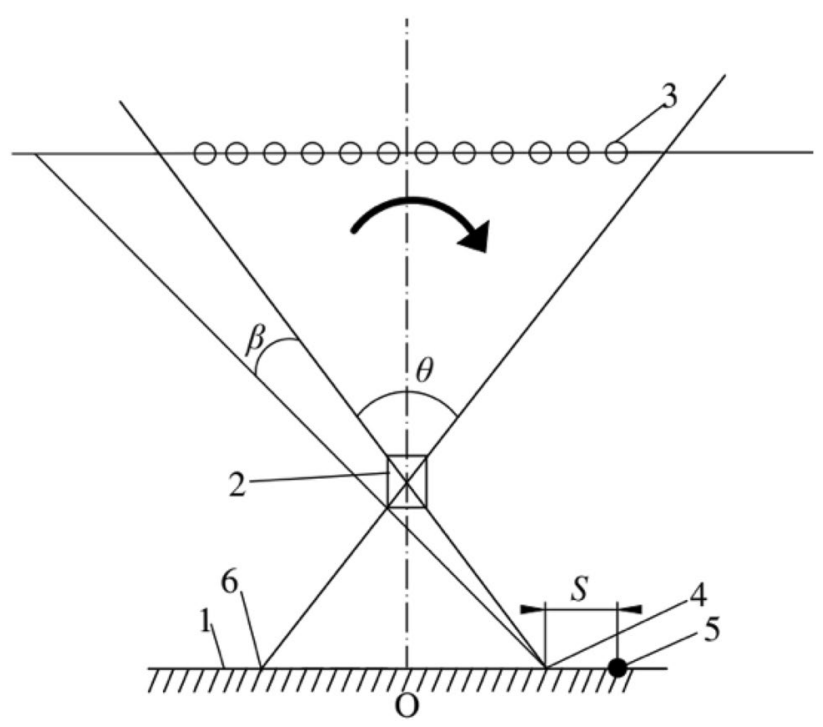

Fig. 5 Schematic diagram of furnace tube scanning process. 1: guide rail; 2: watching-fire hole; 3 : furnace tube; 4 : scanning starting point; 5: magnet; 6 : scanning end point 
tinues to move an initial distance $S$ until it arrives at the scanning starting point. Immediately, the rotatory drive motor actuates the rotator to turn the thermodetector counterclockwise until the baffle is detected by the optoelectronic switch. Then, the rotator continues to rotate clockwise at an initial angle $\beta$ (maximum incident angle).

4. The horizontal drive motor and rotatory drive motor work together to rotate the thermodetector clockwise as it moves left. Thus, the light spot emitted by the thermodetector scans the surface of each tube via the corresponding watching-fire hole.

5. When all the tubes have been scanned, both motors cease and the device arrives at the scanning end point. The process begins again at step two and the system is going to measure temperatures through next watching-fire hole.

\section{Derivation of dual-phase synchronization speed for thermodetector}

In step four above, the speed of the horizontal drive motor and angular speed of the rotary drive motor should satisfy the following relation:

$\omega=\frac{2 V_{1}}{\left(2 H_{1}-2 H_{2}-H_{3}\right)}+\frac{2 V_{1}}{\left(2 H_{2}+H_{3}\right)}$

where $\omega$ is the angular speed of the rotary drive motor, $V_{1}$ is the speed of the horizontal drive motor, $H_{1}$ is the distance between the plane of the guide rail and plane of the furnace tube, $\mathrm{H}_{2}$ is the distance between the plane of the guide rail and plane of the watching-fire hole, and $\mathrm{H}_{3}$ is the depth of the watching-fire hole.

The light spot emitted by the thermodetector executes both linear and rotational motion, therefore, to realize uniform scanning, the emitted light continues moving evenly across the plane of the tubes. The key to realizing the above motion is controlling the position and speed of the emitted light.

From Fig. 6, given the speed $V_{1}$ of horizontal drive motor and taking the plane of tubes as the reference system, to maintain the movement of emitted light within the plane, the rotator must have a certain rotational speed $V_{2}$. The relationship between $V_{1}$ and $V_{2}$ can be calculated as:

$V_{2}=\frac{V_{1}}{\cos \left(\frac{\theta}{2}\right)}$,

where $\theta$ is the temperature measurement angle.

Moreover, to ensure the thermodetector scans the surface of each tube evenly, another speed component $V_{3}$ must be

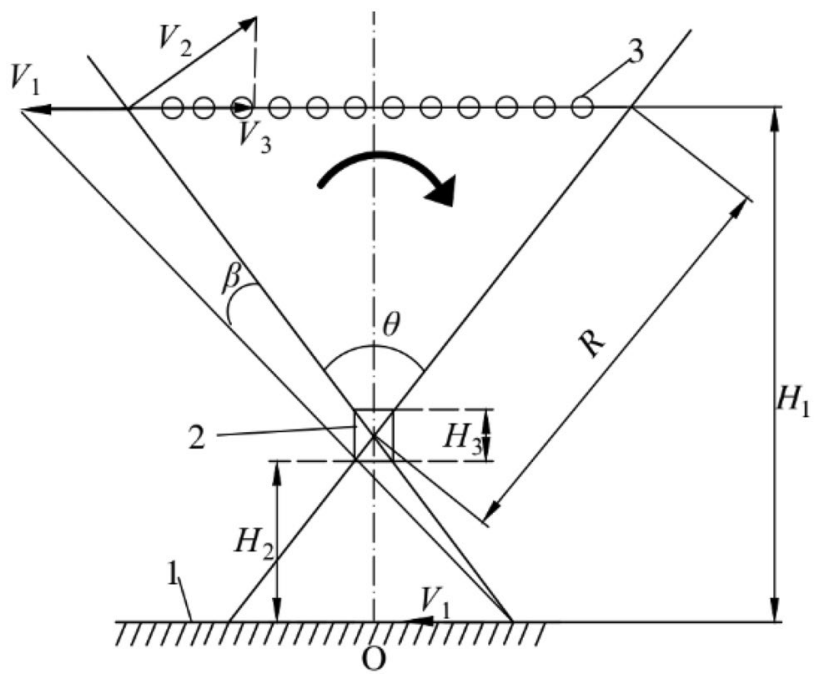

Fig. 6 Relationship between horizontal drive motor speed and rotary drive motor angular speed. 1: guide rail; 2 : watching-fire hole; 3 : furnace tube

added to the rotator. The relationship between $V_{1}$ and $V_{3}$ should satisfy the following equation:

$2 \frac{\left(H_{2}+\frac{H_{3}}{2}\right) \times \tan \left(\frac{\theta}{2}\right)}{V_{1}}=\frac{2 R \times \sin \left(\frac{\theta}{2}\right)}{V_{3}}$

Simplifying Eq. (3), we obtain.

$V_{3}=\frac{2 R \times \cos \left(\frac{\theta}{2}\right) \times V_{1}}{\left(2 H_{2}+H_{3}\right)}$,

where $R$ represents the scanning radius, calculated as

$R=\frac{\left(2 H_{1}-2 H_{2}-H_{3}\right)}{2 \cos \left(\frac{\theta}{2}\right)}$

Combining Eqs. (2) and (4), the angular speed $\omega$ of the rotatory drive motor can be calculated as:

$\omega=\frac{\left(2 R \times \cos \left(\frac{\theta}{2}\right) \times \frac{V_{1}}{\left(2 H_{2}+H_{3}\right)}+V_{1}\right)}{\left(\cos \left(\frac{\theta}{2}\right) \times R\right)}$.

Combining Eqs. (5) and (6), $\omega$ can be further simplified into Eq. (1).

To clarify, stepper motors are used as the horizontal and rotatory drive motors in the temperature measurement drive platform. Measuring $H_{1}, H_{2}$ and $H_{3}$ manually and given the movement speed $V_{1}, \omega$ can be determined using Eq. (1). Next, according to the relationship between speed, impulse 
frequency, and microstepping driver parameter of the stepper motor, $V_{1}$ and $\omega$ can be translated into the impulse frequency required for the stepper motor, thus realizing synchronous control of the temperature measurement platform and rotator.

It should be noted that the system does not need to be calibrated daily because based on the above analysis, once the values of $\beta, H_{1}, H_{2}, H_{3}$ and $V_{1}$ are obtained from the field situation and experience, the maximum incident angle, moving speed of the drive platform, and rotatory speed of the thermodetector can also be determined. Therefore, the system automatically measures the tube surface temperatures and results obtained by the system are stable. This also means that provided the industrial environment does not change, the values of $\beta, H_{1}, H_{2}, H_{3}$ and $V_{1}$ do not change, and there is no need to adjust the system.

\section{Data processing algorithm for thermodetector}

The collected temperature and distance data must be processed by the thermodetector to obtain the final furnace tube surface temperature. Data processing includes initialization, filtering, and filtering correction of the data, and finally, the temperature can be calculated.

\section{Data initialization}

Since different watching-fire holes correspond to different valid furnace tube distances (distance from tube to thermodetector), the valid tube distance $[\alpha, \beta]$ should be initialized according to its corresponding watching-fire hole. The thermodetector also measures distance apart from temperature, which can be used to identify whether temperatures belong to the tube surface or furnace wall. It is worth noting that the thermodetector usually measures all tubes of a hole once before moving to the next operation. Therefore, the temperature and distance data are first stored in the arrays Temp[] and Dist[], respectively. Moreover, some other parameters must be initialized including the overlapping threshold $\gamma$ of the tubes, threshold number of collection points $a$ and $b$, and number of collection points $d_{1}, d_{2}$ and $d_{3}$, which need to be deleted.

\section{Data filtering}

Comparing distances with valid ranges for the tube, it is easy to recognize whether the measured object is the tube or furnace wall, and subsequently select only tube surface temperatures. Hence, for each array of elements, Temp [ $i]$ and Dist $[i]$, the data filtering process is carried out, as shown in Fig. 7.

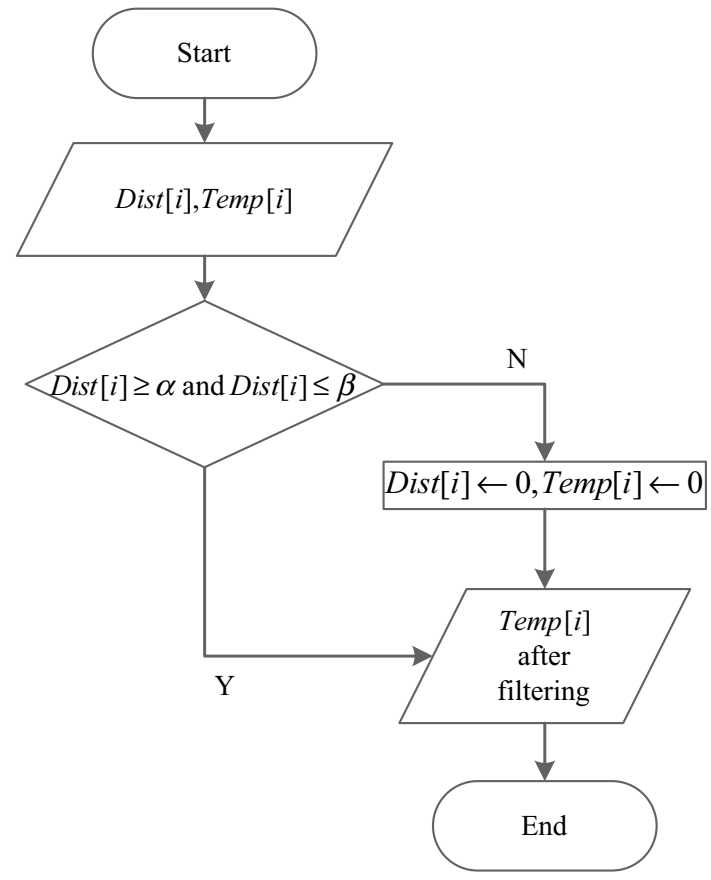

Fig. 7 Data filtering

\section{Filtering correction}

Filtering correction is used to determine indexes corresponding to the start and end collection points of each tube, called the beginning temperature index and ending temperature index. Furthermore, filtering can also be used to deal with overlap of tubes. The filter correction procedure of each tube is shown in Fig. 8.

Based on the filtering process, non-zero elements in the array Temp[] include all tube temperatures, and temperatures of different tubes are separated by a variable number of zeros. Thus, it is easy to determine the beginning and ending temperature indexes of a tube according to these characteristics.

\section{Temperature calculation}

Temperatures of measurement point on the edge of the furnace tube are generally higher or lower than those in the middle area and vary a lot; however, temperatures in the middle area tend to be similar and remain stable. Therefore, temperatures measured in the middle area of the tube are a truer representation of the tube surface temperature. Based on this, the temperatures measured on the edge of the tube are filtered out before calculating the surface temperature of the tube. The temperature calculation procedure is shown in Fig. 9. 


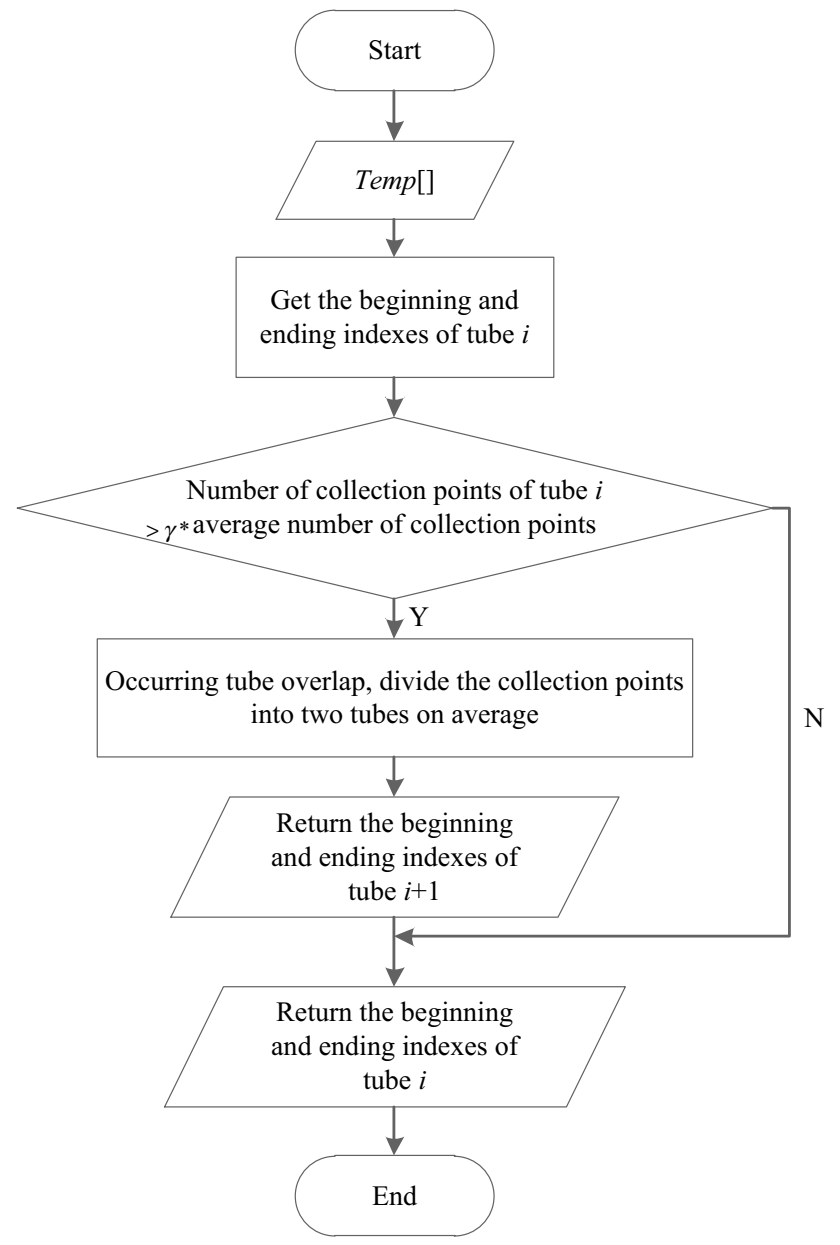

Fig. 8 Filtering correction

\section{System test verification}

The temperature measurement system proposed in this paper was applied to an ethylene plant cracking furnace of a large petrochemical company. The ethylene plant has several cracking furnaces, each of which has eight watchingfire holes and 96 furnace tubes, therefore, 12 tubes can be observed through each hole. To test the effectiveness of the proposed method, two aspects of real measurement experiments were carried out and the results were analyzed.

\section{Analysis of temperature distribution of furnace tube surface}

Through the watching-fire hole five, distances and temperatures of particular points on tubes four and five were measured, and the results are presented as distance and temperature distribution diagrams, as shown in Figs. 10 and 11, respectively. For more precise data, please refer to Tables 1 and 2 of the appendix.
Head and tail points are assumed to be collected from the furnace wall since the distances of these points exceed the valid range, as shown in Figs. 10 and 11, and the remaining points represent measurements on the furnace tube surface. Analyzing the above data, we find that:

(1) In most cases, the temperatures on the edges of the tube are higher than those in the middle area; moreover, the thermodetector scans from the left to the right demonstrating that the temperature of the tube gradually decreases from the edge to middle and then increases from middle to the edge, as seen in Fig. 10 and Table 1. Furthermore, it is apparent that temperatures in the middle area change very little. The reason for the above phenomena is that temperatures of the edge are more easily influenced by thermal radiation from the furnace wall than those in the middle, thus resulting in higher temperatures.

(2) Sometimes, however, a situation occurs in which the temperatures in the middle area are higher than those on the edge, as shown in Fig. 11 and Table 2. Temperatures on the edge (points 12-14) are lower than those in the middle area (points 6-11) because there is a watching-fire hole piston near tube five, which radiates a small amount of heat causing the temperatures on the right edge of the tube to be lower.

Consequently, when calculating the temperature of the tube surface, only temperatures measured in the middle area should be taken into account and averaged to obtain the final result. Here, the final temperature of tube four is the average temperature of collection points 5-9 and the temperature of tube five is calculated by averaging temperatures of points 6-10.

\section{Comparison with manual temperature measurement method}

Measurements were carried out on 12 tubes through watching-fire hole five over 1 week using either the automatic measurement method with our proposed system or the manual measurement way, both using the same infrared temperature measurement instrument. Data curves of temperature change over time were plotted for each experiment, shown in Figs. 12 and 13 (only including tubes 1-6). Moreover, the standard deviation of each tube temperature during this period was calculated, and a graph of the standard deviation distribution is shown in Fig. 14. Exact values of each point on the graphs are listed in Tables 3 and 4 of the appendix.

Analyzing the above data, it can be seen that:

(1) Results obtained using the two methods were similar. The biggest differences were observed between normal 
Fig. 9 Temperature calculation procedure

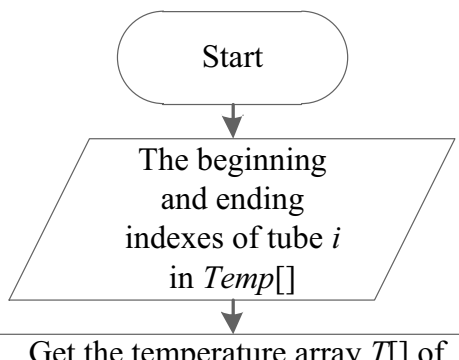

Get the temperature array $T[]$ of tube $i$ from Temp [] and count the number of collection points

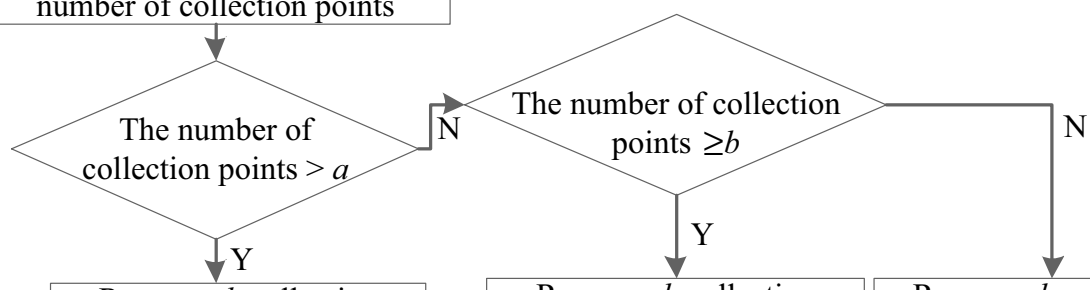

Remove $d_{1}$ collection points at both ends of $T[]$ and average the remaining points as the temperature for tube $i$
Remove $d_{2}$ collection points at both ends of $T[]$ and average the remaining points as the temperature for tube $i$

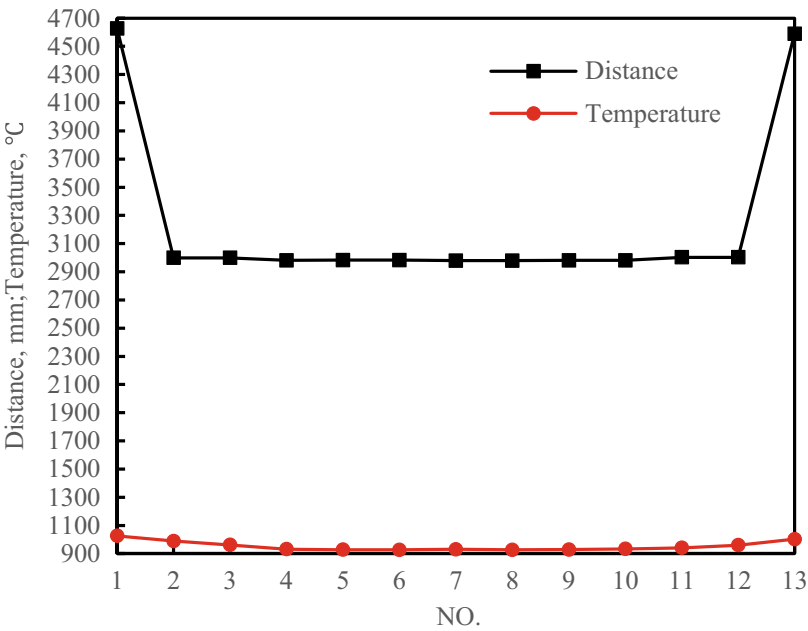

Fig. 10 Distance and temperature distribution of collection points on tube four

temperatures, those gathered from the middle area of the tube surface (points not marked with a red circle in the figures and not marked with * in tables). Differences do not exceed $6{ }^{\circ} \mathrm{C}$ and the trends of curves for both temperature and standard deviation are essentially

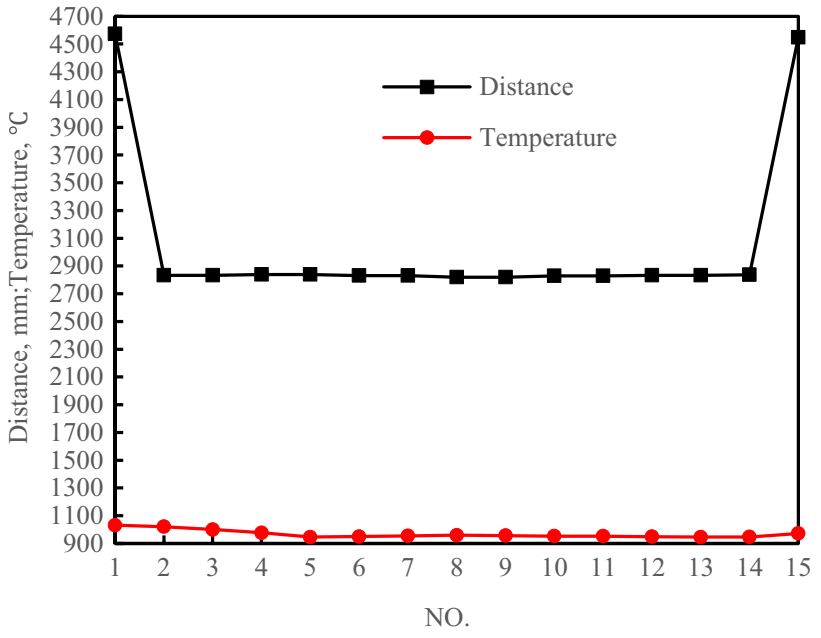

Fig. 11 Distance and temperature distribution of collection points on tube five

the same; however, temperatures obtained using the automatic system are more stable and standard deviations are also smaller.

(2) The automatic method effectively avoids abnormal temperatures at the edges of the tube or furnace wall. 
Table 1 Collection data of tube four

\begin{tabular}{lll}
\hline No. & Distance $(\mathrm{mm})$ & Temperature $\left({ }^{\circ} \mathrm{C}\right)$ \\
\hline 1 & 4626 & 1026 \\
2 & 2999 & 989 \\
3 & 2999 & 962 \\
4 & 2981 & 931 \\
5 & 2983 & 928 \\
6 & 2983 & 927 \\
7 & 2979 & 930 \\
8 & 2979 & 927 \\
9 & 2981 & 929 \\
10 & 2981 & 933 \\
11 & 3003 & 941 \\
12 & 3003 & 960 \\
13 & 4589 & 1002 \\
\hline
\end{tabular}

Table 2 Collection data of tube five

\begin{tabular}{lll}
\hline No. & Distance $(\mathrm{mm})$ & Temperature $\left({ }^{\circ} \mathrm{C}\right)$ \\
\hline 1 & 4573 & 1031 \\
2 & 2833 & 1021 \\
3 & 2833 & 1000 \\
4 & 2839 & 977 \\
5 & 2839 & 946 \\
6 & 2831 & 950 \\
7 & 2831 & 954 \\
8 & 2819 & 959 \\
9 & 2819 & 956 \\
10 & 2829 & 953 \\
11 & 2829 & 953 \\
12 & 2833 & 949 \\
13 & 2833 & 946 \\
14 & 2837 & 947 \\
15 & 4549 & 972 \\
\hline
\end{tabular}

The proposed dual-phase drive synchronization method automatically measures the temperatures of multiple collection points of a tube via the thermodetector and provides enough data points to perform the temperature calculation. Moreover, the temperature processing algorithm easily filters out abnormal temperatures and takes the average of all normal temperatures as the final result, thus reflecting the actual temperature more precisely. However, due to the hostile measurement environment, in addition to human error, the manual method usually collects only one point per tube, which is not always located in the middle area, and therefore, may include abnormal temperatures. For example, the temperature of $945^{\circ} \mathrm{C}$ measured on tube four (Fig. 13) is abnormal since it is higher than the temperatures

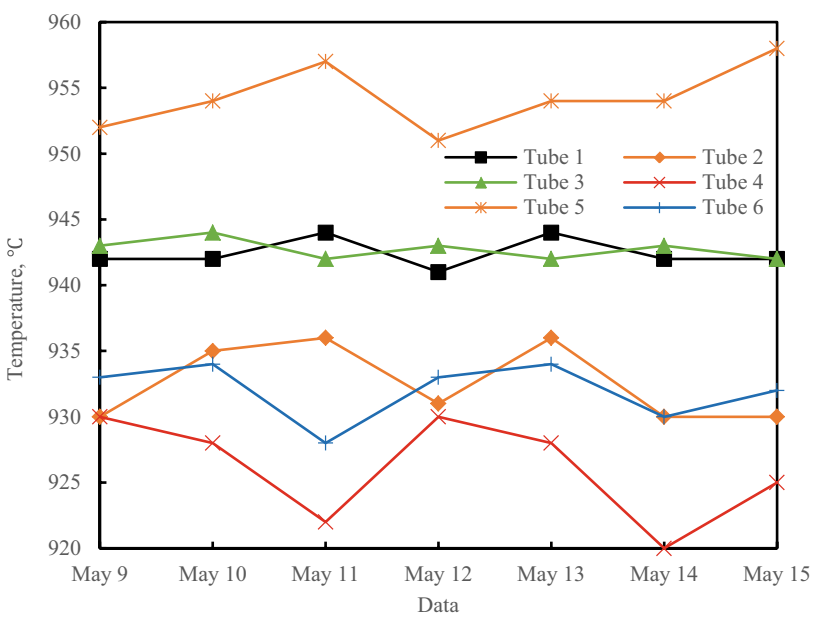

Fig. 12 Temperature distribution obtained using automatic temperature measurement system

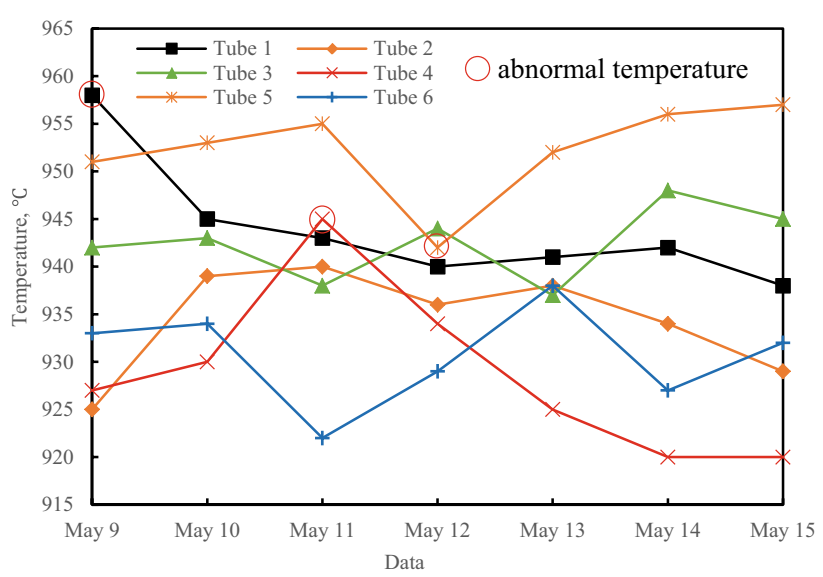

Fig. 13 Temperature distribution obtained using manual temperature measurement method

measured in the middle area and was most likely collected from the edge of the tube. Also, the temperature of $942{ }^{\circ} \mathrm{C}$ measured on tube five (Fig. 13) is an abnormal temperature since it is lower than temperatures measured in the middle area and may have been collected near the piston of the watching-fire hole.

(3) Abnormal temperatures occurring in the manual method are usually hard to detect since there is often not much deviation from normal temperatures; however, the automatic method filters out the abnormal temperatures from the start thus avoiding this phenomenon.

Therefore, the dual-phase drive synchronization method and temperature processing algorithm proposed in this 


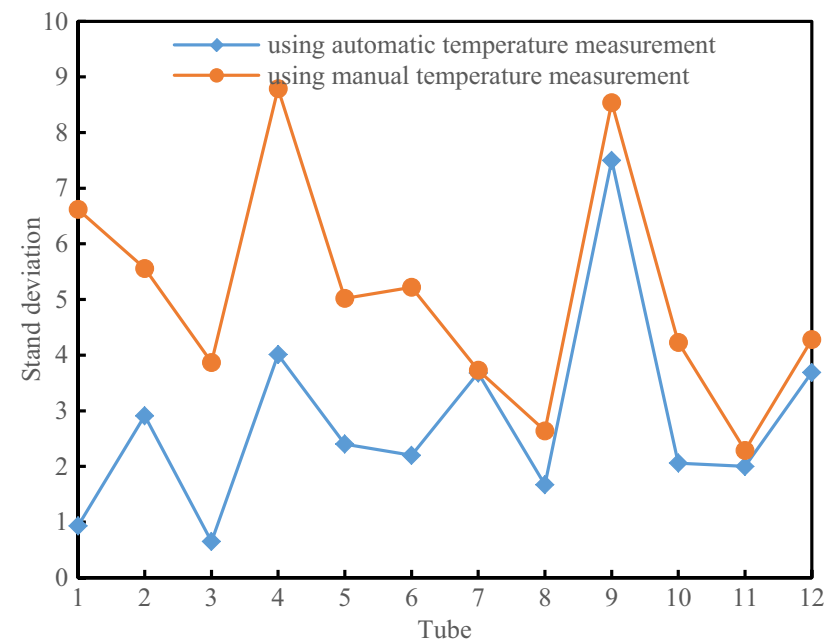

Fig. 14 Distribution of tube temperature standard deviation for automatic versus manual methods paper are effective and avoid the disadvantages of manual temperature measurement method.

\section{Conclusions}

In this paper an external surface temperature measurement system for ethylene cracking furnace tubes was proposed. The system realizes both a dual-phase drive synchronization method and temperature processing algorithm. The dualphase drive synchronization method enables the thermodetector to automatically measure tube temperatures in batches and measure the temperature of a single tube from multiple points and angles, thereby providing numerous data points for more accurate analysis and calculation of temperatures. Furthermore, the temperature processing algorithm allows the thermodetector to differentiate between temperatures of the furnace tube and furnace wall, filter out abnormal temperatures, select high-quality temperatures, and determine the final result. Comparing the results to actual temperature
Table 3 Temperature data obtained using automatic temperature measurement system
Table 4 Temperature data obtained using manual temperature measurement method

\begin{tabular}{lllllllll}
\hline${ }^{\circ} \mathrm{C}$ & May 9 & May 10 & May 11 & May 12 & May 13 & May 14 & May 15 & Stand deviation \\
\hline Tube 1 & 942 & 942 & 944 & 941 & 944 & 942 & 942 & 0.93 \\
Tube 2 & 930 & 935 & 936 & 931 & 936 & 930 & 930 & 2.91 \\
Tube 3 & 943 & 944 & 942 & 943 & 942 & 943 & 942 & 0.65 \\
Tube 4 & 930 & 928 & 922 & 930 & 928 & 920 & 925 & 4.01 \\
Tube 5 & 952 & 954 & 957 & 951 & 954 & 954 & 958 & 2.40 \\
Tube 6 & 933 & 934 & 928 & 933 & 934 & 930 & 932 & 2.20 \\
Tube 7 & 952 & 948 & 944 & 952 & 952 & 944 & 952 & 3.68 \\
Tube 8 & 955 & 956 & 951 & 955 & 955 & 956 & 955 & 1.67 \\
Tube 9 & 944 & 946 & 928 & 943 & 944 & 946 & 930 & 7.50 \\
Tube 10 & 950 & 950 & 946 & 951 & 950 & 950 & 946 & 2.06 \\
Tube 11 & 930 & 931 & 927 & 931 & 930 & 931 & 927 & 2.00 \\
Tube 12 & 967 & 968 & 958 & 968 & 967 & 968 & 965 & 3.69 \\
\hline
\end{tabular}

\begin{tabular}{lllllllll}
\hline${ }^{\circ} \mathrm{C}$ & May 9 & May 10 & May 11 & May 12 & May 13 & May 14 & May 15 & Stand deviation \\
\hline Tube 1 & $958^{*}$ & 945 & 943 & 940 & 941 & 942 & 938 & 6.62 \\
Tube 2 & 925 & 939 & 940 & 936 & 938 & 934 & 929 & 5.56 \\
Tube 3 & 942 & 943 & 938 & 944 & 937 & 948 & 945 & 3.87 \\
Tube 4 & 927 & 930 & $945^{*}$ & 934 & 925 & 920 & 920 & 8.79 \\
Tube 5 & 951 & 953 & 955 & $942 *$ & 952 & 956 & 957 & 5.02 \\
Tube 6 & 933 & 934 & 922 & 929 & 938 & 927 & 932 & 5.22 \\
Tube 7 & 950 & 946 & 946 & 952 & 954 & 946 & 954 & 3.73 \\
Tube 8 & 956 & 955 & 954 & 959 & 952 & 955 & 951 & 2.64 \\
Tube 9 & 939 & 946 & 925 & 938 & 947 & 947 & 931 & 8.54 \\
Tube 10 & 948 & 953 & 946 & 955 & 954 & 954 & 945 & 4.23 \\
Tube 11 & 926 & 928 & 927 & 933 & 928 & 927 & 929 & 2.29 \\
Tube 12 & 968 & 970 & 962 & 969 & 967 & 970 & 959 & 4.28 \\
\hline
\end{tabular}

*Represents abnormal temperature 
measurements of an ethylene cracking furnace tube, the effectiveness of the automatic temperature measurement system and measurement method was demonstrated. Moreover, measurements were shown to be more accurate and stable than traditional manual method, thus reducing costs and human resources. Moreover, successfully applying this system will provide a basis for tube temperature measurements in other petrochemical industry applications.

Acknowledgements This research was financially supported by the National Natural Science Foundation of China (No. 61772145, 61672174), Maoming city science and technology project (No. 2017287) and the Talent Introduction Project of Guangdong University of Petrochemical Technology (No. 2016rc02). We also sincerely thank Junfeng Zhao for providing the helpful suggestions to improve the quality of this paper.

Open Access This article is distributed under the terms of the Creative Commons Attribution 4.0 International License (http://creativeco mmons.org/licenses/by/4.0/), which permits unrestricted use, distribution, and reproduction in any medium, provided you give appropriate credit to the original author(s) and the source, provide a link to the Creative Commons license, and indicate if changes were made.

\section{References}

1. Khodamorad SH, Fatmehsari DH, Rezaie H, Sadeghipour A (2012) Analysis of ethylene cracking furnace tubes. Eng Fail Anal 21:1-8

2. Pregowski P, Goleniewski G, Komosa W, Zwolenik S (2005) Applications of dynamic IR thermography in studying operation of heaters. Proc SPIE 5782:83-92

3. Nishiyama Y, Semba H, Ogawa K, Sawaragi Y, Yamadera Y, Kinomura S (2002) A new carburization resistant alloy for ethylene cracking furnace tubes. In: Conference: CORROSION 2002, 7-11 April. Denver, Colorado
4. Geng LY, Gong JM, Qin XY, Shen LM (2012) Modeling of carburization and thermal stress analysis for ethylene cracking furnace tube. Mater Sci Forum 704:1136-1140

5. Han Y, Geng Z, Wang Z, Mu P (2016) Performance analysis and optimal temperature selection of ethylene cracking furnaces: a data envelopment analysis cross-model integrated analytic hierarchy process. J Anal Appl Pyrol 122:35-44

6. Jin YK, Li JL, Du WL, Wang ZL, Qian F (2013) Outlet temperature correlation and prediction of transfer line exchanger in an industrial steam ethylene pyrolysis process. Chinese J Chem Eng 21(4):388-394

7. Zhou HC, Han SD (2003) Simultaneous reconstruction of temperature distribution, absorptivity of wall surface and absorption coefficient of medium in a 2-D furnace system. Int J Heat Mass Tran 46:2645-2653

8. Masoumi ME, Sadrameli SM, Towfighi J, Niaei A (2006) Simulation, optimization and control of a thermal cracking furnace. Energy 31(4):516-527

9. Lou C, Li WH, Zhou HC, Salinas CT (2011) Experimental investigation on simultaneous measurement of temperature distributions and radiative properties in an oil-fired tunnel furnace by radiation analysis. Int J Heat Mass Tran 54:1-8

10. Cheng Q, Zhang X, Wang Z, Zhou H (2014) Simultaneous measurement of three-dimensional temperature distributions and radiative properties based on radiation image processing technology in a gas-fired pilot tubular furnace. Heat Transfer Eng 35(6-8):770-779

11. Zheng S, Zhang X, Qi C, Zhou H (2015) Modeling of heat transfer and pyrolysis reactions in ethylene cracking furnace based on 3-D combustion monitoring. Int J Therm Sci 94:28-36

12. Peng ZP (2015) Temperature discrimination method and measuring device of the furnace tube surface and the furnace wall. China Patent no 201410666748.5

Publisher's note Springer Nature remains neutral with regard to jurisdictional claims in published maps and institutional affiliations. 